\title{
Therapie der akuten diabetischen Stoffwechselentgleisun- gen bei Erwachsenen (Update 2019)
}

\section{Hyperglykämisch-hyperosmolare und ketoazidotische Stoffwechselentgleisung}

\author{
Susanne Kaser · Harald Sourij · Martin Clodi · Bruno Schneeweiß · Anton N. Laggner · Anton Luger
}

Online publiziert: 12. April 2019

(C) Der/die Autor(en) 2018

\begin{abstract}
Zusammenfassung Akute Stoffwechselentgleisungen können für Erwachsene in Abhängigkeit von ihrem Ausmaß lebensbedrohlich sein. Dementsprechend sind eine rasche umfassende Diagnostik und Therapie sowie eine enge Überwachung der Vitalparameter und Laborbefunde erforderlich. Bei der Therapie, die sich bei der ketoazidotischen (DKA) und hyperglykämisch-hyperosmolaren (HHS) Form nicht wesentlich unterscheidet, kommt dem Ausgleich des meist beträchtlichen Flüssigkeitsdefizits mit mehreren Litern
\end{abstract}

\section{S. Kaser}

Department für Innere Medizin 1, Medizinische Universität Innsbruck, Innsbruck, Österreich

Christian Doppler Labor für Insulinresistenz, Medizinische Universität Innsbruck, Innsbruck, Österreich

\section{H. Sourij}

Klinische Abteilung für Endokrinologie und Diabetologie, Universitätsklinik für Innere Medizin, Medizinische Universität Graz, Graz, Österreich

\section{Clodi}

Abteilung für Innere Medizin, Krankenhaus Barmherzige Brüder Linz, Linz, Österreich

\section{B. Schneeweiß}

Abteilung für Innere Medizin, Landeskrankenhaus Kirchdorf, Kirchdorf, Österreich

\section{A. N. Laggner}

Universitätsklinik für Notfallmedizin, Medizinische Universität Wien, Wien, Österreich

\footnotetext{
A. Luger $(\bowtie)$

Klinische Abteilung für Endokrinologie und Stoffwechsel, Universitätsklinik für Innere Medizin III, Medizinische Universität Wien, Währinger Gürtel 18-20, 1090 Wien, Österreich

anton.luger@meduniwien.ac.at
}

einer physiologischen kristalloiden Lösung eine vorrangige Rolle zu. Bei den Elektrolyten ist insbesondere auf eine ausgeglichene Serum-Kalium-Konzentration zu achten. Normal-Insulin oder rasch wirksame Analoga können initial als i.v.-Bolus verabreicht werden, in der Folge jedoch kontinuierlich über einen Perfusor. Die Umstellung auf eine subkutane Insulintherapie soll erst bei ausgeglichenem Säure-Basen-Haushalt und zufriedenstellender Glykämie erfolgen.

Schlüsselwörter Diabetische Ketoazidose - Hyperglykämisch-hyperosmolare Stoffwechselentgleisung • Serum-Osmolalität · Anionenlücke · Pseudohyponatriämie

Treatment of acute diabetic metabolic crises in adults (Update 2019)

Hyperglycemic hyperosmolar state and ketoacidotic metabolic disorders

Summary Diabetic ketoacidosis (DKA) and the hyperglycemic hyperosmolar state (HHS) represent potentially life-threatening situations in adults. Therefore, rapid comprehensive diagnostic and therapeutic measures with close monitoring of vital and laboratory parameters are required. The treatment of DKA and HHS is essentially the same and replacement of the mostly substantial fluid deficit with several liters of a physiological crystalloid solution is the first and most important step. Serum potassium concentrations need to be carefully monitored to guide its substitution. Regular insulin or rapid acting insulin analogues can be initially administered as an i.v. bolus followed by continuous infusion. Insulin should be switched to subcutaneous injections only after correc- 
tion of the acidosis and stable glucose concentrations within an acceptable range.

Keywords Diabetic ketoacidosis - Hyperglycemic hyperosmolar state - Serum osmolality · Anion gap · Pseudohyponatremia

Ketoazidotische Stoffwechselentgleisungen (DKA) sind wesentlich häufiger als hyperglykämisch-hyperosmolare (HHS). Erstere betreffen zu zwei Dritteln Typ-1-Diabetiker und überwiegend jüngere Patienten im Alter von 18 bis 44 Jahren [1]. Die HHS tritt im Gegensatz dazu viel häufiger bei Typ-2-Diabetikern auf und ist auch aufgrund des höheren Alters des betroffenen Kollektivs und der damit assoziierten häufigeren Komorbiditäten mit einer deutlich höheren Mortalität verbunden (weniger als $1 \%$ bei der DKA und 5-20\% bei der HHS) [1, 2].

\section{Pathogenese, klinisches Bild und Befunde}

Die Unterteilung der diabetischen Stoffwechselentgleisungen in DKA und HHS muss als grob schematisch betrachtet werden, da die DKA stets mit einer Azidose, oft aber nur mit einer mäßiggradigen Hyperglykämie und umgekehrt die HHS mit einem sehr hohen Blutzuckerwert, aber einer meist nur gering ausgeprägten Azidose verbunden ist (Tab. 1). Die DKA ist durch absolutes Fehlen von Insulin gekennzeichnet und infolgedessen fehlender Unterdrückung der Lipolyse und Verwertung freier Fettsäuren. Bei der HHS steht im Gegensatz dazu ein relativer Insulinmangel mit gesteigerter Glukoneogenese und beeinträchtigter peripherer Glukoseverwertung im Vordergrund. Im klinischen Alltag bestehen trotzdem weitgehend Überschneidungen, die HHS ist meist durch einen $\mathrm{pH}>7,3$ und ein Bikarbonat von $>18 \mathrm{mmol} / \mathrm{l}$ gekennzeichnet (Tab. 1). Aggravierend für die Ausbildung der Stoffwechselentgleisung sind zudem die Dehydrierung und der damit verbundene Anstieg der gegenregulatorischen Stresshormone Adrenalin, Noradrenalin und Kortisol [3-6]. Stets sollte an eine möglicherweise auslösende Ursache der Stoffwechselentgleisung gedacht werden, häufig ein Infekt, und eine ggf. erforderliche Diagnostik veranlasst werden. Weitere Ursachen für eine DKA sind häufig die Erstmanifestation eines Diabetes mellitus Typ 1, unterlassene Insulinapplikation oder Insulinpumpendefekte. Insbesondere bei der DKA kann die manchmal bestehende Pseudoperitonitis eine Herausforderung darstellen und muss bei der Indikationseinstellung für eine Laparotomie unbedingt berücksichtigt werden.

Für das Auftreten einer Bewusstseinstrübung bzw. eines Komas ist vorwiegend die Hyperosmolalität verantwortlich. Bei einer Serumosmolarität $<320 \mathrm{mmol} / 1$ muss jedenfalls nach einer anderen Ursache für das Vorliegen des Komas gesucht werden, die ja auch die Ursache für die Stoffwechselentgleisung darstel-
Tab. 1 Laborbefunde bei DKA - HHS. (Adaptiert nach [1, 2])

\begin{tabular}{|l|l|l|}
\hline & DKA & HHS \\
\hline Plasmaglukose $(\mathrm{mg} / \mathrm{dl})$ & $>250$ & $>600$ \\
\hline Arterieller $\mathrm{pH}$ & $<7-7,3$ & $>7,3$ \\
\hline Serumbikarbonat $(\mathrm{mmol} / \mathrm{l})$ & $<10-18$ & $>18$ \\
\hline Harnketone & Positiv & Negativ oder schwach positiv \\
\hline Serumketone $(\mathrm{mmol} / \mathrm{l})$ & $3->8$ & $<0,6$ \\
\hline Effektive Serumosmolalität & Variabel & $>320$ \\
\hline Anionenlücke & $>10$ & Variabel \\
\hline Bewusstseinslage & Klar-Koma & Stupor-Koma \\
\hline
\end{tabular}

Tab. 2 Typische Defizite von Flüssigkeit und Elektrolyten bei DKA+HHS. (Nach [1, 2])

\begin{tabular}{|l|l|l|}
\hline & DKA & HHS \\
\hline Flüssigkeit $(\mathrm{l})$ & 6 & 9 \\
\hline $\mathrm{H}_{2} \mathrm{O}(\mathrm{ml} / \mathrm{kg})$ & 100 & $100-200$ \\
\hline $\mathrm{Na}(\mathrm{mmol} / \mathrm{kg})$ & $7-10$ & $5-13$ \\
\hline $\mathrm{Cl}(\mathrm{mmol} / \mathrm{kg})$ & $3-5$ & $5-15$ \\
\hline $\mathrm{K}(\mathrm{mmol} / \mathrm{kg})$ & $3-5$ & $4-6$ \\
\hline $\mathrm{PO}{ }_{4}(\mathrm{mmol} / \mathrm{kg})$ & $5-7$ & $3-7$ \\
\hline $\mathrm{Mg}(\mathrm{mmol} / \mathrm{kg})$ & $1-2$ & $1-2$ \\
\hline $\mathrm{Ca}(\mathrm{mmol} / \mathrm{kg})$ & $1-2$ & $1-2$ \\
\hline
\end{tabular}

len kann. Für die Beurteilung der Serumosmolalität ist die errechnete von größerer Bedeutung als die gemessene, wobei hier die Formel

$$
\begin{aligned}
& \text { Serumosmolalität }(\mathrm{mmol} / \mathrm{l})= \\
& \qquad \begin{aligned}
2 \times \mathrm{Na}^{+}(\mathrm{mmol} / \mathrm{l})+\mathrm{K}^{+}(\mathrm{mmol} / \mathrm{l}) \\
+ \text { Glukose }(\mathrm{mg} / \mathrm{dl}): 18
\end{aligned}
\end{aligned}
$$

heranzuziehen ist, da BUN ungehindert zwischen Extra- und Intrazellulärraum diffundieren kann und in diesem Fall nicht berücksichtigt werden muss.

Die Anionenlücke, die sich anhand der Formel

$$
\begin{aligned}
\text { Anionenlücke }= & \mathrm{Na}^{+}(\mathrm{mmol} / \mathrm{l}) \\
& -\left(\mathrm{Cl}^{-}(\mathrm{mmol} / \mathrm{l})+\mathrm{HCO}_{3}^{-}(\mathrm{mmol} / \mathrm{l})\right)
\end{aligned}
$$

errechnet [7-10], ist bei der DKA aufgrund der Erhöhung der Ketone ebenfalls vergrößert. Dabei ist zu berücksichtigen, dass die Anionenlücke auch bei Urämie, durch Salicylate, Methylalkohol/Äthanol, Aldehyde, bei Laktatazidose und durch $\ddot{A}(E)$ thylenglycol vergrößert ist (Merkwort: KUSMALE).

$\mathrm{Zu}$ beachten ist ferner, dass in Abhängigkeit vom Ausmaß der Hyperglykämie auch eine Pseudohyponatriämie vorliegen kann: Pro $100 \mathrm{mg} / \mathrm{dl}$ über den Normbereich erhöhte Blutzuckerkonzentration ist die gemessene Serum-Natrium-Konzentration um $1,6 \mathrm{mmol} / 1$ niedriger [1]. 
Tab. 3 Elektrolytkonzentrationen verschiedener Infusionslösungen (im Vergleich zu Plasma)

\begin{tabular}{|c|c|c|c|c|c|c|}
\hline & Plasma & $0,9 \% \mathrm{NaCl}$ & Elo-Mel ${ }^{\circledR}$ isoton & KADC & Ringer & Ringer-Laktat \\
\hline $\mathrm{Na}$ & 141 & 154 & 140 & 90 & 147 & 131 \\
\hline K & 4,5 & - & 5 & 25 & 4 & 5,4 \\
\hline $\mathrm{Ca}$ & 2,5 & - & 2,5 & 1,0 & 2,2 & 1,8 \\
\hline $\mathrm{PO}_{4}$ & - & - & - & 10 & - & - \\
\hline Mg & 1,25 & - & 1,5 & 1,5 & - & 1 \\
\hline $\mathrm{Cl}$ & 103 & 154 & 108 & 65 & 155 & 112 \\
\hline $\mathrm{HCO}_{3}$ & 24 & - & Acetat 45 & Malat 23 & - & Laktat 28 \\
\hline Osmolalität & 280-295 & 308 & 302 & 215 & 309 & 278 \\
\hline
\end{tabular}

Tab. 4 Dosierungsschema für die kontinuierliche Insulinverabreichung mittels Perfusor. Zur Vorbereitung eines Perfusors werden $0,5 \mathrm{ml}$ einer $10 \mathrm{ml}$-Durchstechflasche eines rasch wirksamen Insulinanalogs $\left(\right.$ Apidra ${ }^{\circledR}$, Humalog ${ }^{\circledR}$, NovoRapid ${ }^{\circledR}$, FiAsp $^{\circledR} ; 100 \mathrm{E} / \mathrm{ml}$ ) zu 49,5 ml 0,9\% NaCl zugegeben. $1 \mathrm{ml}$ dieser Infusionslösung entspricht somit $1 \mathrm{E}$ des Insulinanalogs

\begin{tabular}{|c|c|}
\hline Blutzuckerwerte & Insulindosis \\
\hline$<80 \mathrm{mg} / \mathrm{dl}$ & $\begin{array}{l}\text { Pause, Kontrolle nach einer halben } \\
\text { Stunde }\end{array}$ \\
\hline $81-120 \mathrm{mg} / \mathrm{dl}$ & $0,7 \mathrm{ml} / \mathrm{h}(0,7 \mathrm{E} / \mathrm{h})$ \\
\hline $121-150 \mathrm{mg} / \mathrm{dl}$ & $1 \mathrm{ml} / \mathrm{h}(1 \mathrm{E} / \mathrm{h})$ \\
\hline $151-180 \mathrm{mg} / \mathrm{dl}$ & $1,5 \mathrm{ml} / \mathrm{h}(1,5 \mathrm{E} / \mathrm{h})$ \\
\hline $181-210 \mathrm{mg} / \mathrm{dl}$ & $2 \mathrm{ml} / \mathrm{h}(2 \mathrm{E} / \mathrm{h})$ \\
\hline $211-240 \mathrm{mg} / \mathrm{dl}$ & $2,5 \mathrm{ml} / \mathrm{h}(2,5 \mathrm{E} / \mathrm{h})$ \\
\hline $241-270 \mathrm{mg} / \mathrm{dl}$ & $3 \mathrm{ml} / \mathrm{h}(3 \mathrm{E} / \mathrm{h})$ \\
\hline $271-300 \mathrm{mg} / \mathrm{dl}$ & $3,5 \mathrm{ml} / \mathrm{h}(3,5 \mathrm{E} / \mathrm{h})$ \\
\hline $301-330 \mathrm{mg} / \mathrm{dl}$ & $4 \mathrm{ml} / \mathrm{h}(4 \mathrm{E} / \mathrm{h})$ \\
\hline $331-360 \mathrm{mg} / \mathrm{dl}$ & $4,5 \mathrm{ml} / \mathrm{h}(4,5 \mathrm{E} / \mathrm{h})$ \\
\hline $361-390 \mathrm{mg} / \mathrm{dl}$ & $5 \mathrm{ml} / \mathrm{h}(5 \mathrm{E} / \mathrm{h})$ \\
\hline $391-420 \mathrm{mg} / \mathrm{dl}$ & $5,5 \mathrm{ml} / \mathrm{h}(5,5 \mathrm{E} / \mathrm{h})$ \\
\hline $421-450 \mathrm{mg} / \mathrm{dl}$ & $6 \mathrm{ml} / \mathrm{h}(6 \mathrm{E} / \mathrm{h})$ \\
\hline \multicolumn{2}{|c|}{$\begin{array}{l}\text { Bei Werten über } 450 \mathrm{mg} / \mathrm{dl} \text { kann eine Insulindosis von } 8 \mathrm{E} / \mathrm{h} \text { gewählt wer- } \\
\text { den, darüber hinausgehende Dosen allenfalls in besonderen Ausnahmesi } \\
\text { tuationen } \\
\text { N. B.: Sobald ein Patient/eine Patientin essen darf, sollten zusätzlich } \\
\text { präprandial 1-1,5E Insulinanalogon/BE verabreicht werden }\end{array}$} \\
\hline
\end{tabular}

\section{Therapie}

Für die Therapie von vordringlicher Bedeutung ist zunächst der Ausgleich des Flüssigkeitsdefizits, da Insulin sonst nicht wirken kann. Durch die Hyperglykämie kommt es zu einer osmotischen Diurese, die zunächst durch eine „Autotransfusion“ von Flüssigkeit aus dem Intrazellulär- in den Extrazellulärraum ausgeglichen wird. Sie bleibt so lange bestehen, wie die Hyperglykämie besteht. Durchschnittliche Flüssigkeits- und Elektrolytdefizite bei DKA und HHS sind in Tab. 2 angeführt.

In zahlreichen Publikationen wird eine Flüssigkeitssubstitution primär mit 0,9\% Kochsalzlösung empfohlen [1, 4, 5, 8-12]. Aufgrund der unphysiologischen Zusammensetzung (Natriumkonzentration $154 \mathrm{mmol} / \mathrm{l}$, Chloridkonzentration $154 \mathrm{mmol} / \mathrm{l}$ ) be- steht aus pathophysiologischer Sicht aber das Risiko einer Verschlechterung der Azidose durch eine hyperchlorämische Azidose [13]. Besser geeignet für die Flüssigkeitssubstitution scheinen daher kristalloide Lösungen mit physiologischerer Zusammensetzung z.B. Elo-Mel ${ }^{\circledR}$ isoton (Tab. 3) zu sein. In der Regel sollte bei schweren Stoffwechselentgleisungen in der ersten halben Stunde bis Stunde 11 Flüssigkeit verabreicht werden und auch in den weiteren $1 \frac{1 / 2}{\text { bis }}$ $2 \mathrm{~h}$ jeweils etwa 11 pro Stunde in Abhängigkeit von Hydratation, Diurese, Körpergewicht, kardialer Funktion und Elektrolyten. Falls ein zentraler Venenkatheter vorhanden ist, kann die Flüssigkeitssubstitution nach dem ZVD gegeben werden: <0: $1000 \mathrm{ml} / \mathrm{h}, 0-3$ : $500 \mathrm{ml} / \mathrm{h}, 4-8: 250 \mathrm{ml} / \mathrm{h}, 9-12: 100 \mathrm{ml} / \mathrm{h},>12$ : keine Flüssigkeitsgabe.

Auch auf die Kaliumsubstitution sollte von Anfang an größte Aufmerksamkeit gerichtet sein: Bei Kaliumkonzentrationen unter $3,3 \mathrm{mmol} / 1$ sollten vor Beginn einer Insulintherapie $20-40 \mathrm{mmol}$ Kaliummalat (oder alternativ Kaliumchlorid) verabreicht werden, da sonst eine Aggravierung der Hypokaliämie durch den Shift von Kalium mit Insulin und Glukose von extrazellulär nach intrazellulär und durch den Ausgleich der Azidose verursacht werden kann und die Patienten einer zusätzlichen Gefährdung ausgesetzt werden. Bei Kaliumwerten zwischen 3,3 und 5,3 mmol/1 sollten $20 \mathrm{mmol}$ Kaliummalat $\mathrm{zu}$ jedem Liter Flüssigkeit zugegeben werden. Lediglich bei initialen Kaliumkonzentrationen von $>5,3 \mathrm{mmol} / \mathrm{l}$ und deutlichen Hinweisen für eine Hyperkaliämie (EKG: Bradykardie, Knotenrhythmus, hohe, spitze T-Zacken) empfiehlt es sich, zunächst mit der Kaliumsubstitution zuzuwarten [14] und kaliumfreie Elektrolytlösungen zu verwenden.

Insulin, meist ein kurz wirksames Analogon, kann initial als Bolus in einer Dosierung von 6 bis 8 Einheiten (oder $0,1 \mathrm{IE} / \mathrm{kgKG}$ ) i.v. gegeben werden, in der Folge jedoch als kontinuierliche Infusion, die nach aktuellen Blutzuckerwerten angepasst werden muss (Tab. 4). Bei der DKA soll bei Blutzuckerwerten unter $200 \mathrm{mg} / \mathrm{dl}$ und bei der HHS bei Werten unter $250 \mathrm{mg} / \mathrm{dl}$ mit der Infusion einer $5 \%$ Glukoselösung begonnen werden [8], um zu große und abrupte Abfälle der Glukose $\mathrm{zu}$ vermeiden und die weitere Insulininfusion zum Ketoazidoseausgleich ohne Hy- 
poglykämie zu erlauben. Die Gefahr eines Hirnödems durch rasche Blutzuckerabfälle ist vorwiegend bei Kindern gegeben.

Für die Gabe von Bikarbonat besteht keine Evidenz für einen positiven Effekt, es sind auch keine Studien zu erwarten, die den Benefit einer solchen Therapie bei $\mathrm{pH}$-Werten $<7$ nahelegen. Es besteht aber Übereinkunft, dass bei Patienten mit einem $\mathrm{pH}$-Wert $<6,9$ 50-100 mmol Natriumbikarbonat in $200-400 \mathrm{ml} \mathrm{H}_{2} \mathrm{O}$ über $2 \mathrm{~h}$ verabreicht werden sollen [1]. Allerdings sollten die zur Flüssigkeitssubstitution eingesetzten Infusionslösungen ein organisches Salz enthalten, aus welchem Bikarbonat gebildet wird.

Für die Gabe von Phosphat gibt es keine Evidenz für positive Effekte.

Die Therapie von schweren diabetischen Stoffwechselentgleisungen sollte, wenn möglich, zunächst unter Monitoring auf einer Überwachungsstation erfolgen. Blutzuckerwerte müssen anfangs stündlich und im weiteren Verlauf 2- bis 3-stündlich kontrolliert werden, um die Insulindosis anpassen zu können. Auch die Serumelektrolyte Natrium, Kalium, Chlorid und Phosphat ebenso wie Kreatinin, BUN und $\mathrm{pH}$ sollten anfangs in Abhängigkeit von den Ausgangswerten zunächst zumindest 2-stündlich und dann 4-stündlich kontrolliert werden. Da der venöse $\mathrm{pH}$ nur um 0,03 Einheiten niedriger als der arterielle ist, sind venöse Kontrollen des $\mathrm{pH}$ durchaus ausreichend, wenn keine andere Indikation für eine arterielle Blutgasanalyse gegeben ist.

Die Umstellung auf eine subkutane Insulintherapie sollte erst erfolgen, wenn die Azidose ausgeglichen ist und stabile Blutzuckerwerte in einem akzeptablen Bereich vorliegen. Der Ausgleich der Azidose beansprucht mehr Zeit als die Korrektur der Blutzuckerwerte.

Funding Open access funding provided by Medical University of Vienna.

Interessenkonflikt $\mathrm{S}$. Kaser hat von folgenden Unternehmen, die auch fördernde Mitglieder der ÖDG sind, Forschungsunterstützungen und/oder Honorare erhalten: AstraZeneca, Boehringer Ingelheim, Eli Lilly, Merck Sharp \& Dohme, Novartis, Novo Nordisk, Roche, Sanofi-Aventis, Servier, Takeda. H. Sourij hat von folgenden Unternehmen, die auch fördernde Mitglieder der ÖDG sind, Forschungsunterstützungen und/oder Honorare erhalten: Amgen, AstraZeneca, Eli Lilly, Merck Sharp \& Dohme, Novo Nordisk, Sanofi-Aventis, Takeda. M. Clodi hat von folgenden Unternehmen, die auch fördernde Mitglieder der ÖDG sind, Forschungsunterstützungen und/oder Honorare erhalten: AstraZeneca, Boehringer Ingelheim, Eli Lilly, Merck Sharp \& Dohme, Novartis,
Novo Nordisk, Sanofi-Aventis, Servier, Takeda. A. Luger hat von folgenden Unternehmen, die auch fördernde Mitglieder der ÖDG sind, Forschungsunterstützungen und/oder Honorare erhalten: Novo Nordisk, Eli Lilly, Sanofi, Boehringer Ingelheim, MSD, Novartis. B. Schneeweiß und A.N. Laggner geben an, dass kein Interessenkonflikt besteht.

Open Access Dieser Artikel wird unter der Creative Commons Namensnennung 4.0 International Lizenz (http:// creativecommons.org/licenses/by/4.0/deed.de) veröffentlicht, welche die Nutzung, Vervielfältigung, Bearbeitung, Verbreitung und Wiedergabe in jeglichem Medium und Format erlaubt, sofern Sie den/die ursprünglichen Autor(en) und die Quelle ordnungsgemäß nennen, einen Link zur Creative Commons Lizenz beifügen und angeben, ob Änderungen vorgenommen wurden.

\section{Literatur}

1. Kitabchi AE, et al. Hyperglycemic crises in adult patients with diabetes. Diabetes Care. 2009;32(7):1335-43.

2. Kitabchi AE, et al. Hyperglycemic crises in patients with diabetes mellitus. Diabetes Care. 2003;26(Suppl 1):S109-S17.

3. Abrahamian $\mathrm{H}$, et al. Mental disorders and diabetes mellitus. Wien Klin Wochenschr. 2016;128(Suppl2):S170-S8.

4. Schneider S. Hyperglycemic crisis in patients with diabetes mellitus. Med Klin Intensivmed Notfmed. 2012;107(6):435-9.

5. Umpierrez G, Korytkowski M. Diabetic emergencies-ketoacidosis, hyperglycaemic hyperosmolar state and hypoglycaemia. Nat RevEndocrinol.2016;12(4):222-32.

6. Gosmanov AR, et al. Hyperglycemic Crises: Diabetic Ketoacidosis (DKA), And Hyperglycemic Hyperosmolar State (HHS). In:De Groot LJ, et al., Hrsg. Endotext. 2000.

7. Cardoso L, et al. Controversies in the management of hyperglycaemic emergencies in adults with diabetes. Metabolism. 2017;68:43-54.

8. Hirsch IE, et al. Diabetic ketoacidosis and hyperosmolar hyperglycemic state in adults. . UpToDate. 2017.

9. Schumann C, Faust M. Diabetic ketoacidosis and hyperosmolar hyperglycemic state. Dtsch Med Wochenschr. 2018;143(6):384-91.

10. DhatariyaKK, VellankiP.TreatmentofDiabeticKetoacidosis (DKA)/Hyperglycemic Hyperosmolar State (HHS): novel advances in the management of hyperglycemic crises (UK Versus USA). Curr Diab Rep. 2017;17(5):33.

11. Maletkovic J, Drexler A. Diabetic ketoacidosis and hyperglycemic hyperosmolar state. Endocrinol Metab Clin North Am. 2013;42(4):677-95.

12. Van Ness-Otunnu R, Hack JB. Hyperglycemic crisis. JEmerg Med. 2013;45(5):797-805.

13. Tran TTT, et al. Review of evidence for adult diabetic ketoacidosis management protocols. Front Endocrinol (Lausanne). 2017;8:106.

14. Usman A. Initial potassium replacement in diabetic ketoacidosis: the unnoticed area of gap. Front Endocrinol (Lausanne). 2018;9:109. 